\title{
Cardiovascular imaging 2015 in the International Journal of Cardiovascular Imaging
}

\author{
Hiram G. Bezerra ${ }^{1} \cdot$ Ricardo A. Costa $^{2} \cdot$ Johan H. C. Reiber $^{3} \cdot$ Paul Schoenhagen $^{4} \cdot$ \\ Arthur A. Stillman ${ }^{5}$ Johan De Sutter ${ }^{6,7} \cdot$ Nico R. L. Van de Veire ${ }^{6,7}$
}

Published online: 16 April 2016

(C) Springer Science+Business Media Dordrecht 2016

\section{Introduction}

It has become a tradition to provide the annual overview of the published papers in the International Journal of Cardiovascular Imaging in the past year. Please, find below the 2015 overview, which is organized based on the usual imaging modalities. In 2015, the Asian Society of Cardiovascular Imaging again published two ASCI Supplements under the leadership of Adjunct Editor Tae Hoon Kim and his Guest Editors.

\section{X-ray angiography}

Zhang et al. [1] published a paper on the determination of culprit coronary artery branches using hemodynamic indices from angiographic images. This study was performed in 14 anesthetized swine and stenoses were created by inserting plastic tubings in LAD and LCx arterial branches. They used the first-pass distribution analysis (PFA) model to measure absolute coronary blood flow by analyzing the

Johan H. C. Reiber

J.H.C.Reiber@lumc.nl

1 Case Western Reserve University School of Medicine, Cleveland, $\mathrm{OH}$, USA

2 Instituto Dante Pazzanese de Cardiologia, Sao Paulo, Brazil

3 Leiden University Medical Center, Leiden, The Netherlands

4 The Cleveland Clinic Foundation, Cleveland, OH, USA

5 Emory University Hospital, Atlanta, GA, USA

6 AZ Maria Middelares Gent, Gent, Belgium

7 Free University Brussels, Brussels, Belgium propagation of a contrast medium in the coronary system. They concluded that a number of hemodynamic indices could potentially be measured in small coronary arterial branches using this PFA technique from only angiographic images.

Li et al. [2] carried out a study to investigate whether nonculprit coronary lesions in young patients $(<45$ years old $)$ who underwent percutaneous coronary intervention (PCI) with stents have higher rates of atherosclerotic progression than older patients. Eight hundred and forty-eight patients from a single center were enrolled and the progression was determined using 3D QCA; the mean interval between the baseline and FU catheterization was 10.8 months. From the data they could indeed conclude that the non-culprit coronary lesions in these younger patients indeed demonstrate higher rates of atherosclerotic progression.

Three-dimensional rotational angiography (3DRA) is a novel imaging method to guide complex catheter ablations of the left atrium. Wolf et al. [3] investigated the feasibility in visualizing the left ventricle and to develop a protocol for guidance of ventricular tachycardia ablation in 13 patients. They concluded from this small feasibility study that the $3 \mathrm{D}$ reconstruction of the left ventricle is feasible, safe and effective, and that these can be used to guide the ablation procedure; further refinements are foreseen.

Sakaguchi et al. [4] have developed a method for automatically determining the time range when contrast agent flowed from artery to myocardium; this is very important as a step towards measuring myocardial perfusion using coronary X-ray angiographic images. A total of 28 data sets were tested in seven porcine models. The mean length of the optimal time range was $3.61 \pm 1.29$ frames or $2.18 \pm 1.40 \mathrm{~s}$. This method was confirmed to be robust for assessing blood flow measurements in conventional coronary angiography at 15 fps with hand injection of the contrast agent. 
The safety and tolerability of iopromide during cardiac catheterization was tested in Chinese 'real-world' practice in a multi-center setting ( 63 centers) and in a total of 17,513 patients during coronary angiography or PCI [5]. Contrast quality was found to be excellent in $99.1 \%$ of patients. The incidence of adverse drug reaction was very low, could be prevented by pre-medication in at-risk patients.

\section{Nuclear cardiology}

In 2015 different excellent paper in the field of nuclear cardiology were published in the journal. In this review we selected a few papers on the topics of basic science, SPECT/ PET imaging in daily practice and potential new clinical applications of cardiovascular PET/CT imaging.

\section{Basic science}

Cardiac dysfunction and heart failure leads to a decreased organ perfusion which is not easily measured simultaneously. Juarez-Orozco et al. [6] evaluated cardiac and renal perfusion simultaneously with the use of (13) $\mathrm{N}^{-\mathrm{NH}_{3}-}$ microPET in a rat model of myocardial infarction. Ten male Wistar rats underwent sham surgery $(n=5)$ or permanent coronary artery ligation to induce myocardial infarction $(\mathrm{MI}, \mathrm{n}=5)$. Eleven weeks later (13) $\mathrm{N}_{-} \mathrm{NH}_{3}$-microPET was performed. Cardiac perfusion was significantly reduced in the MI group and inversely correlated with MI size. There was also a $17 \%$ non-significant reduction in renal perfusion in the MI group compared to sham with a trend towards greater reduction of perfusion in the cortical regions as compared to the medullar renal regions. Although this study should be considered as preliminary, it shows the ability of (13) $\mathrm{N}-\mathrm{NH}_{3}$-microPET to evaluate cardiac and renal perfusion in a rat model of myocardial infarction.

In a provocative study, Isma'eel et al. [7] investigated the use of artificial neural networks (ANN) to improve risk stratification and prediction of myocardial perfusion imaging (MPI) and angiographic results. They developed ANN models based on the data of 5354 consecutive patients referred from the emergency department for rest-stress MPI after serial negative troponins and normal ECGs. With regards to MPI, their ANN models could reduce testing by $59 \%$ and maintain a $96 \%$ negative predictive value for ruling out ischemia. Application of the ANN models could also avoid $73 \%$ of invasive coronary angiograms, while maintaining a $98 \%$ negative predictive value for detecting obstructive coronary artery disease. An online calculator for clinical use was also created using these ANN models, although the investigators admittedly conclude that further study is needed before the calculator can be used in clinical practice.

\section{Clinical applications of SPECT and PET imaging in daily practice}

Several excellent reviews on the topics of atherosclerosis and multi-modalityimaging were published in 2015. Takx et al. [8] provided an overview of coronary and non-coronary atherosclerosis and summarized the current understanding of different available imaging techniques. The integration of these techniques in clinical practice may allow for superior risk stratification and therapeutic planning and monitoring. Similarly, Ko et al. [9] reviewed the benefits, limitations and imaging findings of various imaging modalities for assessing myocardial perfusion, with particular emphasis on the comparison between nuclear imaging and stress perfusion computed tomography and cardiovascular magnetic resonance imaging.

Perfusion SPECT imaging may be technically challenging in obese patients and data on its prognostic value in the obese are rather scarce. De Lorenzo et al. [10] studied 365 obese patients among 1396 patients referred for single-day rest/stress perfusion imaging using a dedicated multi-pinhole cadmium-zinc telluride (CTZ) SPECT camera. Image quality was good/excellent in $94.5 \%$ of these obese patients. The annualized mortality rates were not significantly different among obese and non-obese patients, being $<1 \%$ with normal CTZ-SPECT and increased with the degree of scan abnormality in both obese and non-obese patients. Obesity itself was not an independent predictor of death. The authors conclude that single-day stress/rest CTZ-SPECT with a multi-pinhole camera provides prognostic information with high image quality in obese patients.

Iron deficiency and cardiac sympathetic impairment play a role in the worsening of heart failure and these 2 conditions may be linked. Nagao et al. [11] evaluated 46 patients with dilated cardiomyopathy with both cardiac T2-MRI using a 3-T scanner for iron deficiency and (123)I-metaiodobenzylguanidine (MIBG) imaging for cardiac sympathetic activity. Myocardial T2 value (M-T2) was calculated by fitting signal intensity data for mid-left ventricular septum to a decay curve and (123)I-MIBG washout rate (MIBG-WR) was calculated using a polar-map technique with tomographic data. Both iron deficiency, expressed by a high M-T2 and MIBG-WR were frequently present and were independent predictors of a worse outcome. The combination of both parameters was a more powerful predictor of adverse outcome than either parameter alone.

\section{Potential new clinical applications of cardiovascular PET/CT imaging}

Positron emission tomography/computed tomography (PET/CT) imaging with (18)F-radiolabeled fluorodeoxyglucose (FDG) is a powerful imaging technique which offers 
new opportunities for cardiovascular imaging. Hetterich et al. [12] evaluated 94 patients without systemic inflammation or steroid medication at baseline and after a mean follow-up of 14.5 months. Calcified plaque volume, lumen area and (18)F-FDG uptake, quantified by the target-to-background ratio (TBR) were measured in the carotid arteries, aorta and iliac arteries. Annualized calcified plaque volume increased by $15.4 \%$, carotid and aortic lumen area decreased by 10.5 and $1.7 \%$ respectively. Of note, there was no significant difference in (18)F-FDG uptake at baseline and follow-up. Hypertension was an independent predictor for total, aortic, and iliac calcified plaque volume progression while carotid lumen reduction was predicted by hypercholesterolemia. In contrast, aortic lumen reduction was associated with BMI and mean (18)F-FDG uptake. This study illustrates that the natural progression of different vascular beds may be influenced by different risk factors and can be monitored over time by FDG-PET/CT imaging.

Inflammation is also associated with the development of atrial fibrillation (AF). Joseph et al. [13] evaluated the relationship between AF, splenic metabolic activity and aortic inflammation by (18)F-FDG PET imaging in 70 subjects: 35 with $\mathrm{AF}$ and 35 matched controls without AF. The results of their study indicate that AF is associated with higher splenic activity (and thus higher hematopoietic tissue activation) as well as higher aorta (18)F-FDG uptake (and thus higher arterial inflammation). These interesting results indicate that more studies are needed to clarify the mechanisms by which this cardio-splenic axis is implicated in AF patients.

Recent studies have shown promising results using (18) F-FDG PET/CT for the diagnosis of prosthetic valve endocarditis (PVE). However, negative controls were usually lacking in these studies. Fagman et al. [14] compared (18) F-FDG uptake around prosthetic aortic valves in 8 patients with definite PVE to the (18)F-FDG uptake in 19 patients with an aortic prosthesis without PVE. Visual analysis showed a sensitivity of $75 \%$, specificity of $87 \%$, positive likelihood ratio of 4.8 and negative likelihood ratio of 0.3 . Semi-quantitative analysis using maximal standardized uptake values (SUV) in the valve area and in the descending aorta showed an area under the curve of $0.90(95 \% \mathrm{CI}$ 0.74-1.0) using ROC-curve analysis for the SUV-ratio. The authors concluded that (18)F-FDG uptake in the prosthetic valve area had an overall good diagnostic performance in the diagnosis of PVE.

Gorla et al. [15] investigated the relationship between (18)F-FDP PET/CT findings and serological biomarkers of inflammation (including measurements of C-reactive protein and D-dimer serum levels) in 60 patients with acute aortic syndromes. An aortic wall pathology was considered PET positive by a maximum standardized uptake value $>2.5$ and this finding was present in $41.7 \%$ of patients. The authors documented an association between pathological glucose uptake in aortic wall lesions and high CRP levels during admission and also an association between pathological glucose uptake and increased mortality and adverse cardiovascular events at 3-year follow-up. The combination of PET results with D-dimer levels had the best discriminant value of cardiovascular events during follow-up.

\section{Echocardiography}

In 2015 several interesting papers were published in the field of cardiac ultrasound. A small selection of these papers from every issue of the International Journal of Cardiovascular Imaging is summarized in the following paragraphs.

\section{Radial artery monitoring by ultrasound after transradial coronary angiography}

Transradial coronary procedures are increasingly being used. Lisowska et al. [16] studied 220 patients with acute coronary syndromes who underwent percutaneous coronary intervention performed via transradial access and studied radial artery's patency immediately after the procedure and in long-term observation using ultrasound technology. Radial artery's occlusion was found in $15 \%$ of the patients immediately after the procedure. In a multifactorial analysis the only factor influencing radial artery patency promptly after the procedure was procedure duration. In the followup radial artery remained still obstructive in $12.7 \%$ of the patients, but the chronic radial artery obstruction was clinically silent. Due to the insignificant frequency of the occurrence of radial artery obstruction after percutaneous intervention in acute coronary syndromes and lack of clinical consequences, the authors do not recommend routine periprocedural radial artery evaluation.

\section{Feasibility of exercise echocardiography to evaluate mitral regurgitation}

Exercise transthoracic echocardiography was recently proposed to help risk stratification of mitral regurgitation. Few data are available on the feasibility of Doppler echocardiographic recordings at exercise in daily practice in both secondary and primary mitral regurgitation. In this study [17] comprehensive resting and exercise echocardiography were performed in 72 unselected patients with at least moderate primary or secondary mitral regurgitation in two French university hospitals. Monitoring of the contractile reserve and systolic pulmonary artery pressure appeared informative and less challenging than mitral regurgitation quantification using the PISA method. Monitoring of effective regurgitant orifice was more feasible in ischemic mitral regurgitation than in asymptomatic primary mitral regurgitation. 


\section{Value of strain echocardiography in chest pain patients} presenting at the emergency room

Lee et al. [18] evaluated the usefulness of 2D strain analysis in the assessment of acute chest pain in patients presenting to the emergency department. Patients with known coronary artery disease, ST-elevation myocardial infarction, nonischemic cardiomyopathy and non-cardiac chest pain were excluded. Echocardiography included visual assessment of regional wall motion abnormality and analysis of global and regional longitudinal strain. The diagnosis of coronary artery disease and the occurrence of cardiac events during 1 month after emergency department visit were reviewed. Compared to wall motion analysis regional longitudinal strain showed higher sensitivity with similar diagnostic accuracy for coronary artery disease. Regional longitudinal strain also demonstrated better diagnostic performance than either global longitudinal strain or pretest probability. Regional longitudinal strain had the highest predictive value for 1-month cardiac events. Strain echocardiography appears to be effective in diagnosing coronary artery disease and predicting future eventswith high sensitivity and negative predictive value in acute chest pain patients visiting the emergency department.

\section{Effects of hypoxic exposure}

Hypoxic exposure has been associated with indices of increased cardiac performance in animals and humans. Dedobbeleer et al. [19] explored left ventricular function using speckle tracking echocardiography and sympathetic tone by spectral analysis of heart rate variability in recently adapted lowlanders versus adapted or maladapted highlanders at high altitude. Short-term altitude exposure in lowlanders alters indices of left ventricular systolic function and increases sympathetic nervous system tone. Life-long altitude exposure in highlanders is associated with similar sympathetic hyperactivity, but preserved parameters of left ventricular function, whereas diastolic function may be altered in those with chronic mountain sickness. Altered left ventricular systolic function in recently acclimatized lowlanders may be explained by combined effects of hypoxia and changes in loading conditions.

\section{Hand-held echocardiography for pre-operative evaluation}

Hand-held echocardiography could be considered an alternative to standard echocardiography in the pre-operative evaluation of patients undergoing non-cardiac surgery. Cavalli et al. [20] compared standard echo to bedside evaluation with a pocket-size imaging device in 100 patients scheduled for non-cardiac surgery. Hand-held echo pro- vided similar information but it was faster and earlier performed compared to standard echocardiography.

\section{Semi-automated echocardiographic evaluation of the right ventricle}

Echocardiographic assessment of the right ventricle is complex. Medvedofsky et al. [21] tested a new approach for automated measurements of right ventricular size and function using speckle tracking by comparing them to the conventional manual methodology. The following parameters were studied in 149 patients with a wide range of right ventricular size and function: end-diastolic and end-systolic areas, fractional area change, dimensions, TAPSE and peak systolic velocity. Time required for the automated analysis was $30 \mathrm{~s}$ per patient compared to $4 \mathrm{~min}$ for conventional analysis. There was a good agreement between the parameters obtained with the semi-automated approach and the manual measurements.

\section{Prognostic value of strain in AL amyloidosis with cardiac involvement}

Relative apical sparing of longitudinal systolic strain is a typical echocardiographic feature in AL amyloidosis patients with cardiac involvement. Hu et al. [22] evaluated the change of this typical feature over time by serial echocardiography and its impact on outcome in $24 \mathrm{AL}$ amyloidosis patients with cardiac involvement. Sixteen $(67 \%)$ patients died during a median follow-up of 487 days. Left ventricular global and segmental peak longitudinal strain remained unchanged over time in survivors (all $p>0.05$ ), while left ventricular global, septal-apical and lateral-apical longitudinal systolic strain significantly decreased in non-survivors. The authors concluded that the best followup parameter to predict risk for imminent death is a decrease of longitudinal systolic strain at the lateral apical segment.

\section{Value of stress echocardiography in mild to moderate aortic stenosis}

Patients with mild to moderate aortic stenosis seem to have a worse outcome than commonly expected. Early identification of subjects who may develop a rapid disease progression or cardiovascular events is critical in order to apply adequate risk management. Sonaglioni et al. [23] conducted an observational prospective single-center study to determine the prognostic role of exercise stress echocardiography in 90 patients with mild and moderate asymptomatic aortic stenosis. During the 1-year follow-up time, three patients died, 11 underwent aortic valve replacement and 26 had cardiovascular hospitalizations. $A \geq 15$ exercise-induced increase in the $\mathrm{E} / \mathrm{E}^{\prime}$ ratio (HR 7.69, $\mathrm{p}=0.0001$ ) had a sig- 
nificantly higher risk of cardiac events during the followup. Assessment of diastolic function using Tissue Doppler Imaging during exercise stress echocardiography provides additional prognostic information in such patients.

\section{Magnetic resonance imaging}

There were also interesting advancements in cardiovascular MRI in 2015.

Helck et al. [24] compared atherosclerotic plaque characteristics by MRI in carotid and femoral arteries. They found the maximum percent necrotic core and maximum percent hemorrhage were significantly higher in the carotid arteries compared to the femoral arteries. Maximum percent calcification did not differ significantly. A multicenter reproducibility study for monitoring atherosclerotic plaque progression was presented by Sun et al. [25]. Long latency periods were found to have a significantly negative impact on the image quality of free-breathing coronary wall MRI [26]. Quiescent-interval single-shot (QISS) MRA was evaluated in patients undergoing digital subtraction angiography [27]. Similar diagnostic accuracy in the assessment of PAD was found. A limitation of QISS-MRA was the lower rate of assessable vessel segments compared to contrast enhanced MRA.

Coronary MRA was found to correlate well with stressperfusion MRI for detecting significant CAD and helps exclude CAD in asymptomatic individuals [28]. Extracellular volume $(\mathrm{ECV})$ in patients with chronic total coronary artery occlusion was found to be increased beyond that observed with late gadolinium enhancement (LGE), and correlated with the LV regional wall motion abnormality, which appears to reflect diffuse myocardial fibrosis [29]. A comparison of semi-automated scar quantification techniques using high-resolution, 3-dimensional late-gadolinium enhancement magnetic resonance imaging was examined by Rajchl et al. [30]. They found that while several approaches show acceptable reproducibility, full width at half maximum (FWHM) technique appears superior due to maintenance of accuracy of manual segmentations. Tao et al. [31] demonstrated the feasibility of preprocedural MRI integration into the VT ablation procedure, with highly automated image analysis workflow and minimal mapping effort. Do et al. [32] found that MRI demonstrated that microemboli in moderately ischemic myocardium, but not solely ischemia, prolonged ventricular dysfunction, created perfusion deficits, poor infarct resorption and enhanced compensatory hypertrophy, while moderate ischemia alone caused minor LV changes. Berry et al. [33] found that Strain-encoded CMR with Displacement ENcoding with Stimulated Echoes (DENSE) has the potential to provide clinically useful information on contractility and its recovery over time in patients with acute MI. A multi-parametric score measuring wall motion score index, scar transmurality and extension, was found to better identify increased cardiac remodeling after a coronary event [34]. Right ventricular dysfunction was found to be an independent and incremental predictor of cardiac deaths late after acute myocardial infarction [35]. A closed-chest small animal model was proposed to study the effects of single or repeated coronary occlusion/reperfusion in real-time [36].

Nucifora et al. [37] investigated the relations between myocardial mechanics and the extent of hypertrophy and fibrosis in hypertrophic cardiomyopathy. They found that the extent of hypertrophy and replacement fibrosis influence LV systolic mechanics while extent of replacement fibrosis and interstitial fibrosis influence LV diastolic mechanics. Basal infarct location but not larger infarct size was found to be associated with a successful outcome after alcohol septal ablation in patients with hypertrophic obstructive cardiomyopathy [38]. The ECV was found to be greater in patients with dilated cardiomyopathy without LGE than controls [39]. Rider et al. found that myocardial tissue phase mapping reveals impaired myocardial tissue velocities in obesity [40]. The combination of cardiac T2* MRI and MIBG scintigraphy was a more powerful predictor of MACE than either parameter alone [41]. Zhang et al. [42] showed that myocardial T2 mapping provides evidence for subclinical myocardial inflammation in patients with systemic lubus erthematosus.

Cole et al. [43] investigated the reproducibility of left ventricular function evaluated by qualitative grading and by eyeball estimation of ejection fraction (EF) techniques, and how they are affected by image quality, experience and accreditation. They found that the reproducibility of visual grading of LV function and LVEF estimation is dependent on image quality, but individuals cannot themselves identify when poor image quality isdisrupting their LV function estimate. A 3D cine balanced steady state free precession technique allows for accurate single breath-hold volumetric cine CMR and enables substantial improvements in scanner time efficiency without sacrificing diagnostic accuracy [44]. After surgical ventricular remodeling after myocardial infarction the two ventricles were found to show functional recoupling [45]. Polte et al. [46] compared the 'standard' method [left ventricular stroke volume (LVSV)-aortic forward flow (AoFF)], 'volumetric' [LVSVright ventricular stroke volume (RVSV)] and 'flow' method [mitral inflow (MiIF)AoFF] in patients with mitral regurgitation. Mitral regurgitant volumes (MRVs)/ fractions (MRFs) were larger (p $\backslash 0.0001)$ using the 'standard' method $(90 \pm 31 \mathrm{ml} / 51 \pm 11 \%)$ compared with the 'volumetric' $(76 \pm 30 \mathrm{ml} / 42 \pm 11 \%)$ and 'flow' method ( $70 \pm 32 \mathrm{ml} / 44 \pm 15 \%)$. Inter-observer variability was lowest for the 'flow' and highest for the 'volumetric' method, 
while intra-observer variability was similar for all three methods. Gorter et al. [47] found that the Pulmonary regurgitant volume is superior to fraction using background-corrected phase contrast MRI in determining the severity of regurgitation in repaired tetralogy of Fallot. Noninvasive 4D pressure difference mapping derived from 4D flow MRI enables detection of altered intraluminal aortic pressures and showed significant spatial and temporal changes in patients with repaired aortic coarctation [48]. Myocardial $\mathrm{T} 1$ and T2 mapping in diastolic and systolic phase were compared by Tessa et al. [49]. They found that acquisition in systole increases the number of evaluable segments. Phase contrast flow imaging was found to benefit from the administration of an intravascular contrast agent by improving the delineation of the perfused lumen and reducing phase noise in flow measurements [50]. An accelerated self-gated ultrashort echo time pulse sequence was evaluated for functional imaging of murine hearts [51].

Hwang et al. [52] evaluated the relation between the left atrial wall composition by late gadolinium enhancement cardiac magnetic resonance and the complex fractioned atrial electrogram (CFAE) in patients with persistent atrial fibrillation (PeAF). They found that the evaluation of LA wall normal substrate by CMR might be useful to predict the CFAE occurrence before catheter ablation of PeAF. Hwang et al. [53] also compared left atrial late gadolinium enhancement (LA-LGE) quantification methods based on different references to characterize the left atrial wall in patients with atrial fibrillation. They found that the full width at half maximum (FWHM) technique, which was based on the maximum signal from the mitral valve with high signal intensity could be used to provide a reproducible quantification of LA-LGE related to atrial fibrillation.

Considerable variability was found among the current methods for quantifying the arrhythmogenic MRI defined gray zone and scar core [54]. Aquaro et al. [55] found that a multiparametric CMR approach allowed distinction between active inflammation, chronic pericarditis with constriction and effusion without inflammation. Confirmation of the utility of a multiparametric approach was found by Bolen et al. [56] using surgical confirmation. Four septal bounce-derived parameters have a good accuracy in differentiating constrictive pericarditis from volunteers [57].

\section{Computed tomography}

In 2015 the journal has again published a wide range of topics related to cardiovascular computed tomography submitted by authors throughout the world. It is interesting to review some trends in 3 major areas, including established indications of the great vessels, coronary CTA, and CTA in the context of structural and vascular intervention.
Established indications for CTA include assessment of the aorta and pulmonary arteries: the majority of submitted papers are focused on clinical applications rather than technical innovation

DeFreitag et al. [58] describe the value of abdominal aortic aneurysm (AAA) screening in patients with documented thoracic aortic aneurysm (TAA). In a patient population of 462 patients with a TAA, $104(22.5 \%)$ also had an AAA. The strongest correlation was seen in patients with age $>55$, systemic hypertension, smoking history and a TAA in the descending thoracic aorta.

Rodrigues et al. [59] describe the relationship between clot burden by $\mathrm{CT}$ and echocardiographic severity of right ventricular dysfunction in patient with acute pulmonary embolism. RV function was evaluated with fractional area change (FAC), with dysfunction defined by FAC $<40 \%$. In patients with acute PE, the effect of clot burden on RV performance was better expressed by FAC than tissue Doppler indexes.

Schölzel et al. [60] examine the prediction of hemodynamic improvement after pulmonary endarterectomy in chronic thromboembolic pulmonary hypertension. Prior to surgery, pulmonary artery (PA) diameter indices were calculated from chest $\mathrm{CT}$ scans and different echocardiographic measurements to evaluate pulmonary hypertension were obtained. Gender, pre-operative mean PAP, PA diameter indices, and tricuspid annular plane systolic excursion were all predictors for hemodynamic improvement after PEA. The pre-operative PA diameter indexed for body surface area was the only independent predictor for hemodynamic improvement after PEA in CTEPH patients. In all patients who died within 30 days after PEA, persistent pulmonary hypertension was present.

\section{Coronary CTA remains a topic of significant interest: two papers discussed the application of subtraction angiography with $\mathrm{CT}$ in order to reduce limitations in calcified coronary lesions or coronary stents}

Yoshioka et al. [61] describe that in patients with severely calcified lesions, subtraction CTA using a second-generation 320-detector row scanner showed improvement in diagnostic accuracy, compared to conventional CTA reconstruction.

Amanuma et al. [62] provide data demonstrating that subtraction CCTA was associated with significantly higher diagnostic accuracy than conventional CCTA in the evaluation of in-stent restenosis.

Several papers describe different techniques to assess hemodynamic, functional significance of coronary lesions

Pugliese et al. [63] report that a segmental calcium score (SCS), in conjunction with calcification morphology, 
anatomical location, patient's symptoms and clinical history, can be helpful to estimate the probability of $\geq 50 \%$ coronary stenosis.

Linde et al. [64] examined the relationship between epicardial coronary artery stenosis severity and the corresponding regional transmural perfusion at rest and during adenosine stress. The data demonstrate a relationship between semi-quantitative perfusion measurements by MDCT and severity of coronary artery stenoses and Liu et al. [65] compare calculation of fractional flow reserve based on $\mathrm{CT}\left(\mathrm{FFR}_{\mathrm{CTA}}\right)$ and conventional angiography $\left(\mathrm{FFR}_{\mathrm{QCA}}\right)$ based on computational fluid dynamics (CFD) requires reconstruction of patient-specific coronary geometry and estimation of hyperemic flow rate. The authors demonstrate acceptable correlation, but agreement in distinguishing functional significant stenosis was moderate. Disagreement was found in $10(17.5 \%)$ vessels. The data demonstrate that image resolution has a significant impact on FFR computation.

Miller et al. [66] examined heterogeneity of the spatial distribution of arteriolar perfusion territories as a correlate of intra-myocardial microvessels density and distribution. In patient with dilated cardiomyopathy (DCM) and a control group segmental myocardial perfusion (F) and intra-myocardial blood volume $(\mathrm{Bv})$ were computed. No significant differences between the groups were identified in overall myocardial $\mathrm{F}$ or Bv. However, the myocardial regional data showed significantly increased spatial heterogeneity in the DCM group when compared to the control group. The authors conclude that altered function of the sub-resolution intra-myocardial microcirculation can be quantified with myocardial perfusion CT.

\section{CT plays an important role in the context of structural and vascular intervention, where it allows procedural planning and is complementary to echocardiography and angiography. Examples are transcatheter aortic and mitral valve replacement}

Foldyna et al. [67] compared semi-automatic versus manual CT image segmentation prior to transapical aortic valve replacement, with focus on speed and precision of experienced versus inexperienced observers [10]. The data demonstrate excellent inter-software and inter-observer reliability, with a mean ICC of 0.95 .

Von Aspern et al. [68] examined the influence of areabased versus perimeter-based calculation on the effective diameter of the aortic annulus prior to transcatheter aortic valve implantation. Effective diameter measurements derived from the area were significantly smaller compared to perimeter-based measurements (mean difference: systolic $0.72 \pm 0.3 \mathrm{~mm}$ and diastolic $0.81 \pm 0.4 \mathrm{~mm} ; \mathrm{p}<0.001$ ). While the area-based ED was significantly influenced by the cardiac cycle with a mean difference of $0.4 \pm 0.6 \mathrm{~mm}$ $(p=0.009)$, no significant difference was found for the perimeter-based ED (mean difference: $0.2 \pm 0.4 ; p=0.07$ ).

Sündermann et al. [69] describe early experience of computed tomography for planning and postoperative imaging of transvenous mitral annuloplasty in an animal model [12]. Seven domestic pigs underwent $\mathrm{CT}$ before and after implantation of a Cardioband ${ }^{\mathrm{TM}}$ (a percutaneously implantable mitral valve annuloplasty device) with a second-generation 128-section dual-source CT machine. CT visualization and measurement of the mitral valve annulus dimensions were feasible. The authors suggest that CTA will likely become the imaging method of choice for procedure planning of Cardioband ${ }^{\mathrm{TM}}$ implantations or other transcatheter mitral annuloplasty devices.

\section{While the above described papers reflect popular trends, there are other topics beyond the 'mainstream'}

Winther et al. [70] describe a low-cost approach for the diagnosis of CAD, based on detection of diastolic murmurs from post-stenotic coronary turbulence evaluated with an acoustic sensor placed on the chest wall. The authors compared diagnostic accuracy of this acoustic test (CAD-score) to clinical risk stratification and coronary artery calcium score (CACS). Obstructive CAD was defined as more than 50\% diameter stenosis diagnosed by quantitative analysis of the ICA. Diagnostic accuracy evaluated by receiver operating characteristic curves was $72 \%$ for the CAD-score, which was similar to the Diamond-Forrester clinical risk stratification score, $79 \%(\mathrm{p}=0.12)$, but lower than CACS.

Such novel topics may have impact on future development, but sometimes are more difficult to be published. The editors of the journal encourage potential authors to submit such papers.

\section{Intravascular imaging}

The year of 2015 repeated the success of 2014 with high quality papers in intravascular imaging. In almost all issue of The International Journal of Cardiovascular Imaging an interesting paper involving intravascular imaging was present, totalizing 17 papers along the year.

\section{OCT feasibility/technical aspects}

As iOCT continues to evolve and clinical adoption increases, special attention to aspects of imaging acquisition appears in recent publications:

\section{Temporal resolution matters!}

A lot is emphasized on the high spatial resolution of OCT but not much attention is devoted to the high 
temporal resolution of the method. Koyama et al. [71] aimed to clarify the influence of cardiac motion on the accuracy and reproducibility of longitudinal measurements obtained using OCT and to determine the optimal pullback speed. In a very well structured and presented study, various speed from 10 to $40 \mathrm{~mm} / \mathrm{s}$ were tested, all exponentially higher than IVUS figures and all resulting in minimal interobserver variability and very high accuracy. Basically high temporal resolution "freezes" the heart beat motion. The faster the pullback the less is the impact of cardiac motion. The price to pay is less frame density and although 100-200 $\mu \mathrm{m}$ spacing are more than enough, higher spacing results in more interpolation for 3D rendering images. There is very few applications for $3 \mathrm{D}$ rendering today, so my conclusion is; go faster! For the industry we welcome even faster lasers that will ultimately allow faster pullback without compromise on the frame density and interpretation.

\section{Merry-go-round}

Elahi et al. [72] investigated the effect of neointimal thickness and in the appearance of metallic struts in OCT images. Presence of a thick neointima resulted in lateral elongation (merry-go-round) of stent strut features in IVOCT images. This was described before with catheter eccentricity and now another factor appears to directly impact. Awareness of this phenomenon is important to avoid over reaction when interpreting post stent images.

\section{Go fast or go home!}

Suter et al. [73] work helps shed some light on the impact of media selection, flow-rate settings in displacing blood for obtaining diagnostic quality OFDI images. The higher the flow rate up to $4 \mathrm{cc} / \mathrm{s}$, the better is the image quality. This stresses the fact that power injector is still the preferable and most reliable way to obtain intracoronary OCT images.

\section{OCT going molecular!}

Intravascular near-infrared fluorescence (NIRF) imaging is a novel technique for the assessment of molecular processes, e.g. inflammation. It requires individual frame calibration. Ughi et al. [74] developed an algorithm for the automatic distance-correction of dual-modality OCT-NIRF images and concluded that the proposed algorithm enables fully automatic visualization of dual modality OCT-NIRF pullbacks, and provides an accurate and efficient calibration of NIRF data for quantification of the molecular agent in the atherosclerotic vessel wall. We look forward to see first clinical application of this hybrid modality.
Imaging all arteries!

Taniwaki et al. [75] assessed the feasibility and the procedural and long-term safety of intracoronary imaging of all 3 vessels. Two modalities, OCT and IVUS, were attempted in patients with STEMI undergoing primary PCI in the setting of IBIS-4 study. A high success rate $(\sim 80 \%)$ was achieved, with few complications, less than $2.0 \%$. Multi-modality three-vessel intra-vascular imaging in STEMI patients undergoing primary PCI is consistent with a high degree of success and can be performed safely without impact on cardiovascular events at long term follow-up. The inability to image $\sim 20 \%$ of the vessels also highlights the opportunity for the industry to continue to invest in higher performance catheters.

\section{IVUS and OCT for device interrogation}

\section{Platinum versus cobalt}

Kim et al. [76] show lower percent of acute malapposition with Firehawk versus Xience. No difference between the two platforms at follow-up time was noticed. Are the differences in the acute result a consequence of the stent composition or design? Most important, is there any biological effect of acute malapposition?

\section{Yes, IVUS can see and track BVS degradation}

The literature on BVS clearly favors OCT as the primary modality for BVS follow-up. The unmatched resolution and tissue contrast of OCT makes it the natural choice. Interestingly Campos et al. [77] sought to validate IVUS quantitative echogenicity as a surrogate for molecular weight assessment of poly-L-lactide-acid (PLLA) bioresorbable scaffold (Absorb BVS, Abbott Vascular, Santa Clara, CA). The authors relied in an elaborate 9 time points porcine model. IVUS high intensity gray level quantification is correlated to Absorb BVS residual molecular weight and can be used as a surrogate for the monitoring of the degradation of semi-crystalline polymers scaffolds.

\section{Atherosclerosis}

\section{Vulnerable plaque; systemic versus focal problem?}

Aono et al. [78] suggest that the angioscopic progression of aortic atherosclerosis is closely associated with vulnerability and the extent of coronary stenosis, indicating that vulnerability toward atherosclerotic plaque development occurs simultaneously in the coronary tree and systemic arteries. We believe this is another evidence that not only the plaque, but that the patient is also vulnerable. 


\section{Plaque progression}

Kashiyama et al. [79] demonstrated CKD stage 3-5 was an independent predictor of plaque progression. Moderate to advanced CKD was associated with coronary plaque progression characterized by greater lipid and fibrotic plaque volumes, despite statin treatment.

\section{Endothelial dysfunction underlying substrate}

Puri et al. [80] showed that following NSTEMI, both lipidic and necrotic plaque content each are associated with segmental endothelial dysfunction. The link between plaque composition and vessel reactivity provides a mechanistic basis of the pathogenesis associated with endothelial dysfunction.

\section{Angiography is not enough!}

Refaat et al. [81] showed that OCT demonstrated plaque rupture and thrombosis in the majority of ACS patients presenting with complex lesion. One-third of non-complex angiography correlates with ruptured plaque by OCT. This is important evidence that OCT has arole in identifying the culprit lesion particularly in NSTEMI situations.

\section{Is this just spasm?}

Park et al. [82] used OCT to evaluate morphologic changes in vasospastic lesions, which can cause acute coronary syndrome (ACS) or chronic stable variant angina (VA). Maximal intima thickness of spastic segment was significantly greater in the ACS group than in the VA group. In patients with vasospasm-induced ACS, intimal tear, intimal erosion, and micro thrombi are major abnormal morphologic findings of OCT compared with patients with chronic stable VA.

\section{Bright spots}

Minami et al. [83] by means of OCT demonstrated that, using a novel algorithm, a significantly higher density of bright spots in the culprit lesions of patients presenting with ACS. Higher density on plaque rupture patients compared to those presenting with SAP. The density of bright spots also correlates with inflammatory status. These results suggest that the quantitative assessment of bright spot density may be useful in evaluating plaque vulnerability.

\section{IVUS going volumetric}

Sakurai et al. [84] sought to determine the correlation between the fractional flow reserve (FFR) values and volumetric plaque assessment by intravascular ultrasound
(IVUS) parameters derived from classic gray-scale IVUS and integrated backscatter (IB)-IVUS. Lipid plaque volume (LPV) was significantly correlated with the FFR value. It is unclear how to translate this findings clinically, but it is insightful to the fact that we apparently do better when entire volume is taking into account rather than simple cross-section.

\section{Clinical setting}

Imaging the guiding catheter!

Frequently we have guiding catheter imaging within a routine OCT pullback. Is there any useful information there? Scalone et al. [85] sought to detect the frequency of thrombus inside the guiding catheter by OCT and its relationship with clinical and procedural factors. They showed that $60 \%$ of patients had thrombus in the guiding catheter and at multivariate analysis, total procedural time and time between first heparin administration and OCT pullback were only predictors of intra-catheter thrombus. This can be a surrogate of under anticoagulated patient.

\section{Tissue prolapse}

Sohn et al. [86] aimed to compare the detection rate of tissue prolapse (TP) in OCT and IVUS after DES implantation and evaluate clinical implication of TP at 2 years after percutaneous coronary intervention. TP was detected in $95 \%$ of stented lesions by OCT and $45 \%$ of stented lesions by IVUS among 40 stented lesions in 38 patients. The study is however under powered for clinical outcomes.

\section{Call for papers}

Despite robust evidence on the clinical benefit of intravascular imaging, the penetration of these methods on coronary intervention is low worldwide with the exception of Japan. We hope to see more clinical utilization of both IVUS and OCT in the clinical setting and will prioritize papers with clinical application of these methods.

\section{References}

1. Zhang Z, Chen J, Takarada S, Molloi S (2015) Determination of culprit coronary artery branches using hemodynamic indices from angiographic images. Int J Cardiovasc Imaging 31:11-19

2. Li J, Han Y, Jing J, Tu S, Chen W, Reiber JHC, Chen Y (2015) Non-culprit coronary lesions in young patients have higher rates of atherosclerotic progression. Int $\mathrm{J}$ Cardiovasc Imaging 31:889-897

3. Wolf J, Starek Z, Jez J, Lehar F, Lukasova M, Kulik T, Novak M (2015) Rotational angiography of left ventricle to guide ventricular tachycardia ablation. Int J Cardiovasc Imaging 31:899-904 
4. Sakaguchi T, Ichihara T, Natsume T, Yao J, Yousuf O, Trost JC, Lima JAC, George RT (2015) Development of a method for automated and stable myocardial perfusion measurement using coronary X-ray angiographic images. Int J Cardiovasc Imaging 31:905-914

5. Chen J-Y, Liu Y, Zhou Y-L, Tan N, Zhang B, Chen P-Y, Chen L-B (2015) Safety and tolerability of iopromide in patients undergoing cardiac catheterization: real-world multi-center experience with 17,513 patients from the TRUST trial. Int $\mathrm{J}$ Cardiovasc Imaging 31:1281-1291

6. Juarez-Orozco LE, Szymanski MK, Hillege HL, Kruizinga S, Noordzij W, Koole M, Tio RA, Alexanderson E, Dierckx RA, Slart RH (2015) Imaging of cardiac and renal perfusion in a rat model with (13) $\mathrm{N}^{-\mathrm{NH}_{3}}$ micro-PET. Int $\mathrm{J}$ Cardiovasc Imaging 31:213-219

7. Isma'eel HA, Cremer PC, Khalaf S, Almedawar MM, Elhajj IH, Sakr GE, Jaber WA (2016) Artificial neural network modeling enhances risk stratification and can reduce downstream testing for patients with suspected acute coronary syndromes, negative cardiac biomarkers, and normal ECGs. Int J Cardiovasc Imaging 32(4):687-696

8. Takx RA, Partovi S, Ghoshhajra BB (2016) Imaging of atherosclerosis. Int J Cardiovasc Imaging 32(1):5-12

9. Ko SM, Hwang HK, Kim SM, Cho IH (2015) Multi-modality imaging for the assessment of myocardial perfusion with emphasis on stress perfusion $\mathrm{CT}$ and MR imaging. Int $\mathrm{J}$ Cardiovasc Imaging 31(Suppl 1):1-21

10. De Lorenzo A, Peclat T, Amaral AC, Lima RS (2016) Prognostic evaluation in obese patients using a dedicated multipinhole cadmium-zinc telluride SPECT camera. Int J Cardiovasc Imaging 32(2):355-361

11. Nagao M, Baba S, Yonezawa M, Yamasaki Y, Kamitani T, Isoda T, Kawanami S, Maruoka Y, Kitamura Y, Abe K, Higo T, Sunagawa K, Honda H (2015) Prediction of adverse cardiac events in dilated cardiomyopathy using cardiac T2-MRI and MIBG scintigraphy. Int J Cardiovasc Imaging 31(2):399-407

12. Hetterich H, Rominger A, Walter L, Habs M, Volpers S, Hacker M, Reiser MF, Bartenstein P, Saam T (2016) Natural history of atherosclerotic disease progression as assessed by (18)F-FDG PET/CT. Int J Cardiovasc Imaging 32(1):49-59

13. Joseph P, Ishai A, MacNabb M, Abdelbaky A, Lavender ZR, Ruskin J, Nahrendorf M, Tawako A (2016) Atrial fibrillation is associated with hematopoietic tissue activation and arterial inflammation. Int J Cardiovasc Imaging 32(1):113-119

14. Fagman E, van Essen M, Lindqvist F, Snygg-Martin U, BechHanssen O, Svensson G (2016) (18)F-FDG PET/CT in the diagnosis of prosthetic valve endocarditis. Int J Cardiovasc Imaging 32(4):679-686

15. Gorla R, Erbel R, Kuehl H, Kahlert P, Tsagakis K, Jakob G, Mahabadi AA, Schlosser T, Bockisch A, Eggebrecht H, Bossone E, Janosi RA (2015) Prognosticvalue of (18)F-FDG PET/ CT imaging in acute aortic syndromes: comparison with serological biomarkers of inflammation. Int $\mathrm{J}$ Cardiovasc Imaging 31(8):1677-1685

16. Lisowska A, Knapp M, Tycińska A, Sielatycki P, Sawicki R, Kralisz P, Musiał WJ (2015) Radial access during percutaneous interventions in patients with acute coronary syndromes: should we routinely monitor radial artery patency by ultrasonography promptly after the procedure and in long-term observation? Int $\mathbf{J}$ Cardiovasc Imaging 31(1):31-36

17. Coisne A, Levy F, Malaquin D, Richardson M, Quéré JP, Montaigne D, Tribouilloy C (2015) Feasibility of Doppler hemodynamic evaluation of primary and secondary mitral regurgitation during exercise echocardiography. Int J Cardiovasc Imaging 31(2):291-299
18. Lee M, Chang SA, Cho EJ, Park SJ, Choi JO, Lee SC, Oh JK, Park SW (2015) Role of strain values using automated function imaging on transthoracic echocardiography for the assessment of acute chest pain in emergency department. Int J Cardiovasc Imaging 31(3):547-556

19. Dedobbeleer C, Hadefi A, Pichon A, Villafuerte F, Naeije R, Unger P (2015) Left ventricular adaptation to high altitude: speckle tracking echocardiography inlowlanders, healthy highlanders and highlanders with chronic mountain sickness. Int $\mathbf{J}$ Cardiovasc Imaging 31(4):743-752

20. Cavallari I, Mega S, Goffredo C, Patti G, Chello M, Di Sciascio G (2015) Hand-held echocardiography in the setting of preoperative cardiac evaluation of patients undergoing non-cardiac surgery: results from a randomized pilot study. Int J Cardiovasc Imaging 31(5):995-1000

21. Medvedofsky D, Addetia K, Hamilton J, Leon Jimenez J, Lang RM, Mor-Avi V (2015) Semi-automated echocardiographic quantification of right ventricular size and function. Int J Cardiovasc Imaging 31(6):1149-1157

22. Hu K, Liu D, Nordbeck P, Cikes M, Störk S, Kramer B, Gaudron PD, Schneider A, Knop S, Ertl G, Bijnens B, Weidemann F, Herrmann $S$ (2015) Impact of monitoring longitudinal systolic strain changes during serial echocardiography on outcome in patients with AL amyloidosis. Int J Cardiovasc Imaging 31(7):1401-1412

23. Sonaglioni A, Lombardo M, Baravelli M, Trotta G, Sommese C, Anzà C (2015) Exercise stress echocardiography with tissue Doppler imaging in risk stratification of mild to moderate aortic stenosis. Int J Cardiovasc Imaging 31(8):1519-1527

24. Helck A, Bianda N, Cantón G, Yuan C, Hippe DS, Reiser MF, Gallino A, Wyttenbach R, Saam T (2015) Intra-individual comparison of carotid and femoral atherosclerotic plaque features with in vivo MR plaque imaging. Int $J$ Cardiovasc Imaging 31:1611-1618

25. Sun J, Zhao X-Q, Balu N, Hippe DS, Hatsukami TS, Isquith DA, Yamada K, Neradilek MB, Cantón G, Xue Y, Fleg JL, DesvigneNickens P, Klimas MT, Padley RJ, Vassileva MT, Wyman BT, Yuan C (2015) Carotid magnetic resonance imaging for monitoring atherosclerotic plaque progression: a multicenter reproducibility study. Int J Cardiovasc Imaging 31:95-103

26. Lin K, Lloyd-Jones DM, Liu Y, Bi X, Lu B, Li D, Carr JC (2015) The role of latency period in quality management for free-breathing coronary wall MRI. Int J Cardiovasc Imaging 31:621-627

27. Wagner M, Knobloch G, Gielen M, Lauff M-T, Romano V, Hamm B, Kröncke T (2015) Nonenhanced peripheral MR-angiography (MRA) at 3 T: evaluation of quiescent-interval single-shot MRA in patients undergoing digital subtraction angiography. Int J Cardiovasc Imaging 31:841-850

28. Song KD, Kim SM, Choe YH, Jung W, Lee S-C, Chang S-A, Choi YH, Sung J (2015) Integrated cardiac magnetic resonance imaging with coronary magnetic resonance angiography, stressperfusion, and delayed-enhancement imaging for the detection of occult coronary artery disease in asymptomatic individuals. Int $\mathbf{J}$ Cardiovasc Imaging 31(Suppl 1):77-89

29. Chen YY, Zhang WG, Yang S, Yun H, Deng SM, Fu CX, Zeng M-S, Jin H, Guo L (2015) Extracellular volume fraction in coronary chronic total occlusion patients. Int J Cardiovasc Imaging 31:1211-1221

30. Rajchl M, Stirrat J, Goubran M, Yu J, Scholl D, Peters TM, White JA (2015) Comparison of semi-automated scar quantification techniques using high-resolution, 3-dimensional late-gadolinium-enhancement magnetic resonance imaging. Int J Cardiovasc Imaging 31:349-357

31. Tao Q, Piers SRD, Lamb HJ, Zeppenfeld K, van der Geest RJ (2015) Preprocedural magnetic resonance imaging for image- 
guided catheter ablation of scar-related ventricular tachycardia. Int J Cardiovasc Imaging 31:369-377

32. Do L, Wilson MW, Krug R, Hetts SW, Saeed M (2015) MRI monitoring of function, perfusion and viability in microembolized moderately ischemic myocardium. Int J Cardiovasc Imaging $31: 1179-1190$

33. McComb C, Carrick D, McClure JD, Woodward R, Radjenovic A, Foster JE, Berry C (2015) Assessment of the relationships between myocardial contractility and infarct tissue revealed by serial magnetic resonance imaging in patients with acute myocardial infarction. Int J Cardiovasc Imaging 31:1201-1209

34. Palazzuoli A, Beltrami M, Gennari L, Dastidar AG, Nuti R, McAlindon E, Angelini GD, Bucciarelli-Ducci C (2015) The impact of infarct size on regional and global left ventricular systolic function: a cardiac magnetic resonance imaging study. Int $\mathrm{J}$ Cardiovasc Imaging 31:1037-1044

35. Di Bella G, Siciliano V, Aquaro GD, De Marchi D, Rovai D, Carerj S, Molinaro S, Lombardi M, Pingitore A (2015) Right ventricular dysfunction: an independent and incremental predictor of cardiac deaths late after acute myocardial infarction. Int J Cardiovasc Imaging 31:379-387

36. h-Ici DO, Jeuthe S, Dietrich T, Berger F, Kuehne T, Kozerke S, Messroghli DR (2015) Closed-chest small animal model to study myocardial infarction in an MRI environment in real time. Int $\mathbf{J}$ Cardiovasc Imaging 31:115-121

37. Nucifora G, Muser D, Gianfagna P, Morocutti G, Proclemer A (2015) Systolic and diastolic myocardial mechanics in hypertrophic cardiomyopathy and their link to the extent of hypertrophy, replacement fibrosis and interstitial fibrosis. Int $\mathrm{J}$ Cardiovasc Imaging 31:1603-1610

38. Steggerda RC, Geluk CA, Brouwer W, van Rossum AC, Berg ten JM, van den Berg MP (2015) Basal infarct location but not larger infarct size is associated with a successful outcome after alcohol septal ablation in patients with hypertrophic obstructive cardiomyopathy: a cardiovascular magnetic resonance imaging study. Int J Cardiovasc Imaging 31:831-839

39. Hong YJ, Park CH, Kim YJ, Hur J, Lee H-J, Hong SR, Suh YJ, Greiser A, Paek MY, Choi BW, Kim TH (2015) Extracellular volume fraction in dilated cardiomyopathy patients without obvious late gadolinium enhancement: comparison with healthy control subjects. Int J Cardiovasc Imaging 31(Suppl 1):115-122

40. Rider OJ, Ajufo E, Ali MK, Petersen SE, Nethononda R, Francis JM, Neubauer S (2015) Myocardial tissue phase mapping reveals impaired myocardial tissue velocities in obesity. Int J Cardiovasc Imaging 31:339-347

41. Nagao M, Baba S, Yonezawa M, Yamasaki Y, Kamitani T, Isoda T, Kawanami S, Maruoka Y, Kitamura Y, Abe K, Higo T, Sunagawa K, Honda H (2015) Prediction of adverse cardiac events in dilated cardiomyopathy using cardiac T2* MRI and MIBG scintigraphy. Int J Cardiovasc Imaging 31:399-407

42. Zhang Y, Corona-Villalobos CP, Kiani AN, Eng J, Kamel IR, Zimmerman SL, Petri M (2015) Myocardial T2 mapping by cardiovascular magnetic resonance reveals subclinical myocardial inflammation in patients with systemic lupus erythematosus. Int $\mathbf{J}$ Cardiovasc Imaging 31:389-397

43. Cole GD, Dhutia NM, Shun-Shin MJ, Willson K, Harrison J, Raphael CE, Zolgharni M, Mayet J, Francis DP (2015) Defining the real-world reproducibility of visual grading of left ventricular function and visual estimation of left ventricular ejection fraction: impact of image quality, experience and accreditation. Int $\mathrm{J}$ Cardiovasc Imaging 31:1303-1314

44. Jeong D, Schiebler ML, Lai P, Wang K, Vigen KK, François CJ (2015) Single breath hold 3D cardiac cine MRI using kat-ARC: preliminary results at $1.5 \mathrm{~T}$. Int J Cardiovasc Imaging 31:851-857

45. Secchi F, Petrini M, Di Leo G, Bandera F, Castelvecchio S, Guazzi M, Menicanti L, Sardanelli F (2015) Recoupling of right and left ventricle pump function after surgical ventricle restoration: a cardiac magnetic resonance study. Int J Cardiovasc Imaging 31:813-820

46. Polte CL, Bech-Hanssen O, Johnsson Å, Gao SA, Lagerstrand KM (2015) Mitral regurgitation quantification by cardiovascular magnetic resonance: a comparison of indirect quantification methods. Int J Cardiovasc Imaging 31:1223-1231

47. Gorter TM, van Melle JP, Freling HG, Ebels T, Bartelds B, Pieper PG, Berger RMF, van Veldhuisen DJ, Willems TP (2015) Pulmonary regurgitant volume is superior to fraction using background-corrected phase contrast MRI in determining the severity of regurgitation in repaired tetralogy of Fallot. Int J Cardiovasc Imaging 31:1169-1177

48. Rengier F, Delles M, Eichhorn J, Azad Y-J, von Tengg-Kobligk H, Ley-Zaporozhan J, Dillmann R, Kauczor H-U, Unterhinninghofen R, Ley S (2015) Noninvasive 4D pressure difference mapping derived from 4D flow MRI in patients with repaired aortic coarctation: comparison with young healthy volunteers. Int J Cardiovasc Imaging 31:823-830

49. Tessa C, Diciotti S, Landini N, Lilli A, Del Meglio J, Salvatori L, Giannelli M, Greiser A, Vignali C, Casolo G (2015) Myocardial $\mathrm{T} 1$ and $\mathrm{T} 2$ mapping in diastolic and systolic phase. Int J Cardiovasc Imaging 31:1001-1010

50. Makowski MR, Wiethoff AJ, Ebersberger HU, Botnar RM, Razavi R, Schaeffter T, Greil GF (2015) 2D phase contrast blood flow velocity measurements of the thoracic vasculature: comparison of the effect of gadofosveset trisodium and gadopentetate dimeglumine. Int J Cardiovasc Imaging 31:409-416

51. Motaal AG, Noorman N, de Graaf WL, Hoerr V, Florack LMJ, Nicolay K, Strijkers GJ (2015) Functional imaging of murine hearts using accelerated self-gated UTE cine MRI. Int J Cardiovasc Imaging 31:83-94

52. Hwang SH, Oh Y-W, Lee DI, Shim J, Park S-W, Kim Y-H (2015) Relation between left atrial wall composition by late gadolinium enhancement and complex fractionated atrial electrograms in patients with persistent atrial fibrillation: influence of nonfibrotic substrate in the left atrium. Int JCardiovasc Imaging 31:1191-1199

53. Hwang SH, Oh Y-W, Lee DI, Shim J, Park S-W, Kim Y-H (2015) Evaluation of quantification methods for left arial late gadolinium enhancement based on different references in patients with atrial fibrillation. Int $\mathbf{J}$ Cardiovasc Imaging 31(Suppl 1):91-101

54. Mesubi O, Ego-Osuala K, Jeudy J, Purtilo J, Synowski S, Abutaleb A, Niekoop M, Abdulghani M, Asoglu R, See V, Saliaris A, Shorofsky S, Dickfeld T (2015) Differences in quantitative assessment of myocardial scar and gray zone by LGE-CMR imaging using established gray zone protocols. Int J Cardiovasc Imaging 31:359-368

55. Aquaro GD, Barison A, Cagnolo A, Todiere G, Lombardi M, Emdin M (2015) Role of tissue characterization by Cardiac Magnetic Resonance in the diagnosis of constrictive pericarditis. Int $\mathrm{J}$ Cardiovasc Imaging 31:1021-1031

56. Bolen MA, Rajiah P, Kusunose K, Collier P, Klein A, Popovic ZB, Flamm SD, Cardiac MR (2015) Imaging in constrictive pericarditis: multiparametric assessment in patients with surgically proven constriction. Int J Cardiovasc Imaging 31:859-866

57. Angheloiu GO, Rayarao G, Williams R, Yamrozik J, Doyle M, Biederman RWW (2015) Magnetic resonance characterization of septal bounce: findings of blood impact physiology. Int J Cardiovasc Imaging 31:105-113

58. DeFreitas MR, Quint LE, Watcharotone K, Nan B, Ranella MJ, Hider JR, Liu PS, Williams DM, Eliason JL, Patel HJ (2016) Evaluation for abdominal aortic aneurysms is justified in patients with thoracic aortic aneurysms. Int $\mathrm{J}$ Cardiovasc Imaging 32(4):647-653 
59. Rodrigues AC, Guimaraes L, Guimaraes JF, Monaco C, Cordovil A, Lira E, Vieira ML, Fischer CH, Nomura C, Morhy S (2015) Relationship of clot burden and echocardiographic severity of right ventricular dysfunction after acute pulmonary embolism. Int J Cardiovasc Imaging 31(3):509-515

60. Schölzel BE, Post MC, van de Bruaene A, Dymarkowski S, Wuyts W, Meyns B, Budts W, Delcroix M (2015) Prediction of hemodynamic improvement after pulmonary endarterectomy in chronic thromboembolic pulmonary hypertension using noninvasive imaging. Int J Cardiovasc Imaging 31(1):143-150

61. Yoshioka K, Tanaka R, Muranaka K, Sasaki T, Ueda T, Chiba T, Takeda K, Sugawara T (2015) Subtraction coronary CT angiography using second-generation 320-detector row CT. Int J Cardiovasc Imaging 31(Suppl 1):51-58

62. Amanuma M, Kondo T, Sano T, Takayanagi T, Matsutani H, Sekine T, Arai T, Morita H, Ishizaka K, Arakita K, Iwasa A, Takase S (2016) Assessment of coronary in-stent restenosis: value of subtraction coronary computed tomography angiography. Int J Cardiovasc Imaging 32(4):661-670

63. Pugliese F, Hunink MG, Meijboom WB, Gruszczynsnka K, Rengo M, Zou L, Baron I, Dijkshoorn ML, Krestin GP, de Feyter PJ (2015) Computed tomography segmental calcium score (SCS) to predict stenosis severity of calcified coronary lesions. Int J Cardiovasc Imaging 31(8):1663-1675

64. Linde JJ, Kühl JT, Hove JD, Sørgaard M, Kelbæk H, Nielsen WB, Kofoed KF (2015) Transmural myocardial perfusion gradients in relation to coronary artery stenoses severity assessed by cardiac multidetector computed tomography. Int J Cardiovasc Imaging 31(1):171-180

65. Liu L, Yang W, Nagahara Y, Li Y, Lamooki SR, Muramatsu T, Kitslaar P, Sarai M, Ozaki Y, Barlis P, Yan F, Reiber JH, Tu S (2016) The impact of image resolution on computation of fractional flow reserve: coronary computed tomography angiography versus 3-dimensional quantitative coronary angiography. Int $\mathrm{J}$ Cardiovasc Imaging 32(3):513-523

66. Miller WL, Behrenbeck TR, McCollough $\mathrm{CH}$, Williamson EE, Leng S, Kline TL, Ritman EL (2015) Coronary microcirculation changes in non-ischemic dilated cardiomyopathy identified by novel perfusion CT. Int J Cardiovasc Imaging 31(4):881-888

67. Foldyna B, Jungert C, Luecke C, von Aspern K, Boehmer-Lasthaus S, Rueth EM, Grothoff M, Nitzsche S, Gutberlet M, Mohr FW, Lehmkuhl L (2015) CT evaluation prior to transapical aortic valve replacement: semi-automatic versus manual image segmentation. Int J Cardiovasc Imaging 31(6):1233-1242

68. von Aspern K, Foldyna B, Etz CD, Hoyer A, Girrbach F, Holzhey D, Lücke C, Grothoff M, Linke A, Mohr FW, Gutberlet M, Lehmkuhl L (2015) Effective diameter of the aortic annulus prior to transcatheter aortic valve implantation: influence of area-based versus perimeter-based calculation. Int $\mathrm{J}$ Cardiovasc Imaging 31(1):163-169

69. Sündermann SH, Gordic S, Manka R, Cesarovic N, Falk V, Maisano F, Alkadhi H (2015) Computed tomography for planning and postoperative imaging of transvenous mitral annuloplasty: first experience in an animal model. Int $\mathrm{J}$ Cardiovasc Imaging31(1):135-142

70. Winther S, Schmidt SE, Holm NR, Toft E, Struijk JJ, Bøtker HE, Bøttcher M (2016) Diagnosing coronary artery disease by sound analysis from coronary stenosis induced turbulent blood flow: diagnostic performance in patients with stable angina pectoris. Int J Cardiovasc Imaging 32(2):235-245. doi:10.1007/ s10554-015-0753-4

71. Koyama K, Yoneyama K, Mitarai T, Kuwata S, Kongoji K, Harada $\mathrm{T}$ et al (2015) Evaluation of the influence of cardiac motion on the accuracy and reproducibility of longitudinal measurements and the corresponding image quality in optical frequency domain imaging: an ex vivo investigation of the optimal pullback speed. Int J Cardiovasc Imaging 31:1115-1123

72. Elahi S, Ho D, Feldman MD, Dijkstra J, Milner TE (2015) Evaluation of IVOCT imaging of coronary artery metallic stents with neointimal coverage. Int J Cardiovasc Imaging 31:463-470

73. Suter MJ, Kashiwagi M, Gallagher KA, Nadkarni SK, Asanani $\mathrm{N}$, Tanaka A et al (2015) Optimizing flushing parameters in intracoronary optical coherence tomography: an in vivo swine study. Int J Cardiovase Imaging 31:1097-1106

74. Ughi GJ, Verjans J, Fard AM, Wang H, Osborn E, Hara T et al (2015) Dual modality intravascular optical coherence tomography (OCT) and near-infrared fluorescence (NIRF) imaging: a fully automated algorithm for the distance-calibration of NIRF signal intensity for quantitative molecular imaging. Int J Cardiovasc Imaging 31:259-268

75. Taniwaki M, Radu MD, Garcia-Garcia HM, Heg D, Kelbaek $\mathrm{H}$, Holmvang $\mathrm{L}$ et al (2015) Long-term safety and feasibility of three-vessel multimodality intravascular imaging in patients with ST-elevation myocardial infarction: the IBIS-4 (integrated biomarker and imaging study) substudy. Int J Cardiovasc Imaging 31:915-926

76. Kim BK, Shin DH, Kim JS, Ko YG, Choi D, Jang Y et al (2015) Randomized comparison of acute stent malapposition between platinum-chromium versus cobalt-chromium everolimus-eluting stents. Int J Cardiovasc Imaging 31:269-277

77. Campos CM, Ishibashi Y, Eggermont J, Nakatani S, Cho YK, Dijkstra J et al (2015) Echogenicity as a surrogate for bioresorbable everolimus-eluting scaffold degradation:analysis at 1-, 3-, 6-, 12-18, 24-, 30-, 36- and 42-month follow-up in a porcine model. Int J Cardiovasc Imaging 31:471-482

78. Aono J, Ikeda S, Katsumata Y, Higashi H, Ohshima K, Ishibashi $\mathrm{K}$ et al (2015) Correlation between plaque vulnerability of aorta and coronary artery: an evaluation of plaque activity by direct visualization with angioscopy. Int J Cardiovasc Imaging 31:1107-1114

79. Kashiyama K, Sonoda S, Muraoka Y, Suzuki Y, Kamezaki F, Tsuda Y et al (2015) Coronary plaque progression of non-culprit lesions after culprit percutaneous coronary intervention in patients with moderate to advanced chronic kidney disease: intravascular ultrasound and integrated backscatter intravascular ultrasound study. Int J Cardiovasc Imaging 31:935-945

80. Puri R, Nicholls SJ, Brennan DM, Andrews J, Liew GY, Carbone A et al (2015) Coronary atheroma composition and its association with segmental endothelial dysfunction in non-ST segment elevation myocardial infarction: novel insights with radiofrequency (iMAP) intravascular ultrasonography. Int J Cardiovasc Imaging 31:247-257

81. Refaat H, Niccoli G, Gramegna M, Montone RA, Burzotta F, Leone AM et al (2015) Optical coherence tomography features of angiographic complex and smooth lesions in acute coronary syndromes. Int J Cardiovasc Imaging 31:927-934

82. Park HC, Shin JH, Jeong WK, Choi SI, Kim SG (2015) Comparison of morphologic findings obtained by optical coherence tomography in acute coronary syndrome caused by vasospasm and chronic stable variant angina. Int $\mathrm{J}$ Cardiovasc Imaging 31:229-237

83. Minami Y, Phipps JE, Hoyt T, Milner TE, Ong DS, Soeda T et al (2015) Clinical utility of quantitative bright spots analysis in patients with acute coronary syndrome: an optical coherence tomography study. Int J Cardiovasc Imaging 31:1479-1487

84. Sakurai S, Takashima H, Waseda K, Gosho M, Kurita A, Ando $\mathrm{H}$ et al (2015) Influence of plaque characteristics on fractional flow reserve for coronary lesions with intermediate to obstructive stenosis: insights from integrated-backscatter intravascular ultrasound analysis. Int J Cardiovasc Imaging 31(7):1295-1301 
85. Scalone G, Brugaletta S, Garcia-Garcia HM, Martin-Yuste V, Azpeitia Y, Otsuki S et al (2015) Frequency and predictors of thrombus inside the guiding catheter during interventional procedures: an optical coherence tomography study. Int J Cardiovasc Imaging 31:239-246
86. Sohn J,Hur SH, Kim IC, Cho YK, Park HS, Yoon HJ et al (2015) A comparison of tissue prolapse with optical coherence tomography and intravascular ultrasound after drug-eluting stent implantation. Int J Cardiovasc Imaging 31:21-29 